\title{
Colorectal Cancer: Consequences of Chemotherapy Induced Nausea and Vomiting on Patients' Functional Daily Living
}

\author{
Shimaa Shawki Mohammed ${ }^{1}$, Mimi Mohammed Mekkawy ${ }^{2}$, Mohammed Mostafa Wahman ${ }^{3}$, Amna \\ Abdullah Desouky4. \\ 1. B.SC. Nursing, Faculty of Nursing, Assiut University, Assiut, Egypt. \\ 2. Professor, Medical, Surgical Nursing Department, Faculty of Nursing, Assiut University, Assiut, Egypt. \\ 3. Lecturer, Clinical oncology, Faculty of Medicine, Qena University, Qena, Egypt. \\ 4. Lecturer, Medical, Surgical Nursing Department, Faculty of Nursing, Assiut University, Assiut, Egypt.
}

\begin{abstract}
The aim of this study: To assess consequences due to chemotherapy induced nausea and vomiting on patients' functional daily living. Research design: Descriptive design was utilized to conduct this study. Setting: The study was conducted in the internal oncology department at Qena Health Insurance Hospital. Sample: Forty-eight male and female patients admitted in the internal oncology department of the above mentioned hospital. Tools: Tool (I): Patient assessment questionnaire. Tool (II): Functional Living Index for Emesis (FLIE) Questionnaire. Results: About one third of studied patients suffered from consequences in the form of malnutrition, electrolyte imbalance, Mallory_Weiss Syndrome (31.3\%), delayed treatment, salivation and fever, high antiemetic cost, (33.3\%,18.8\%, $20.8 \%$ respectively)which reflected on their functional daily living as $97 \%$ had worse condition before chemotherapy and all patents had worse condition of functional daily living after chemotherapy too. Conclusion: Consequences due to Chemotherapy induced nausea and vomiting had a maximum impact on nearly all patients' functional daily living either before or after chemotherapy. Recommendation: Colorectal cancer patients who receive chemotherapy in need to intensive nursing care and appropriate strategies to manage chemotherapy side effects and consequences related to these side effects which can improve their functional daily living.
\end{abstract}

Key words: Colorectal Cancer, Consequences, Chemotherapy, Nausea \& Vomiting, Functional Daily Living.

\section{Introduction}

Colorectal cancer (CRC) is the development of cancer from the colon or rectum. Globally, CRC is the third most common cancer worldwide after lung and breast cancers. The incidences of CRC in the Arab world and Middle East countries are relatively low. In Egypt, rates of CRC (6.9/105 for males and $5.1 / 105$ for females and making up about $10 \%$ of all cases worldwide (El-Attar, 2015).

Chemotherapy is a type of cancer treatment that includes a combination of medications to treat cancer. also it can be combined with other cancer treatments, such as surgery or radiation therapy. It may be given with a curative or palliative intent, aims to stop or slow the growth of cancer cells over time. It is often given several times over weeks or months as a course of treatment. This course of treatment is made up of a series of treatment periods, called cycle, may be given for one or more days (Pickhardt et al., 2017).

Nausea and vomiting are the two serious side effects of cancer chemotherapy that can cause significant negative impacts on patients' daily activities and on their ability to tolerate and comply with therapy. Around $70-80 \%$ of patients receiving chemotherapy are at risk of CINV, it is categorized according to the timing of its occurrence relative to the administration of chemotherapy into, Acute, delayed, breakthrough and refractory (Cohen et al., 2017).

Uncontrolled CINV can give rise to medical consequences and complication, including poor nutrition, dehydration, electrolyte imbalances, and physical and mental deterioration. In some cases, patients may refuse to continue potentially beneficial treatment regimens because of treatmentassociated uncontrolled CINV, also it requires the use of rescue medication and possible emergency department visits, resulting increasing in the cost of medical care (Hesketh, 2018).

Oncology nurses play a pivotal role in the care of patients receiving chemotherapy and are in a prime position to facilitate better care of patients experiencing chemotherapy-induced nausea and vomiting (Robinson et al., 2018).

\section{Significance of the study}

From the researcher' clinical experience and literature review; it has been observed that most of patients were suffering from chemotherapy induced nausea and vomiting, this increased the probability of exposing them to serious problems or consequences that affect on their functional daily living. So this study would target those patients in 
an attempt to assess the consequences of CINV that can affect on their functional daily living.

\section{Aim of the study}

To assess the consequences of chemotherapy induced nausea and vomiting on functional daily living of colorectal cancer patients .

Research question

What are the consequences of chemotherapy induced nausea and vomiting on functional daily living of colorectal cancer patients ?

\section{Operational definitions}

Consequences: Refer to the effects, results or outcomes to some events.

Chemotherapy-induced nausea and vomiting: An adverse effects of group of chemotherapeutics medications, which is usually self-limited and rarely life-threatening.

Functional daily living (FDL): Refers to patients' activities in the form of physical, emotional, social, and ability to enjoy meals which is measured by using Tool (II).

\section{Patients \& Methods}

Descriptive research design was utilized in this study.

Data collection started in November 2017 and finished in May 2018 at the internal oncology department in Qena Health Insurance Hospital.

\section{Sample}

Forty eight adult patents were collected during six ${ }^{\text {th }}$ months period, these patients diagnosed in stage II or III colorectal cancer and were receiving chemotherapy regimens.

Tools for data collection

\section{Tool (I) :Patient assessment questionnaire}

This sheet was developed by researcher based on national and international literature review to assess socio demographics of studied patient and clinical biomedical data. It consisted of three parts :

\section{Part one: Socio demographic data}

It was developed to assess patients' socio demographic characteristics as name, age, sex, residence, educational level, marital and economic status.

\section{Part two: Medical data}

It included structured items such as past and present heath histories. Which consisted of; disease stages, chemotherapy cycles, types and frequency. Also it included grades of nausea and vomiting before and after chemotherapy.

Part three: Physical examination and diagnostic investigations

This part is concerned with physical examination and diagnostic investigations of studied patients that included nutritional assessment (body surface area \& skin turgor) and laboratory investigation.

Part Four: Consequences of chemotherapy induced nausea and vomiting:

It was developed to assess consequences experienced by studied patients due to CINV, activities that they unable to do due to CINV and actions taken.

Tool (II):Functional Living Index for Emesis (FLIE)Questionnaire (Lindley et al., 2013)

This questionnaire is currently the only validated emesis- and nausea-specific patient-reported outcome which focus on the impact of nausea and vomiting on daily life. The researcher filled the questionnaire from patients by herself, it was administered twice, before chemotherapy administration (FLIE- 1) and on day six after chemotherapy( FLIE-2). It consisted of two domains. Each of them consisted of nine questions. Responses for each item are marked on a $100-\mathrm{mm}$ visual analog scale (VAS) graduated from 1 (the worst condition) to 7 (the best condition) points.

The first question in each domain asks the patient to rate how much nausea (vomiting) he/she had experienced over the past 5 days. The remaining eight questions assess the impact of nausea (vomiting) on the following aspects of a patient's daily life: ability to enjoy meals/liquids, ability to prepare meals/do household tasks, ability to perform daily functions, ability to perform usual recreation/leisure activities, willingness to spend time with family and friends, extent to which the side effect has caused personal hardship and hardship on other.

Scoring system

Each domain score ranges from 9 (maximum impact) to 63 (no impact) with higher scores reflecting less impact on daily life. The impact on daily life was considered separately for nausea and for vomiting. "No impact of nausea (or vomiting) on daily life" was defined as an average item score of $\geq$ 6 on a seven-point scale (domain score of $\geq 54$ ); while, an impact of nausea (or vomiting) on daily life was defined as an average item score of $<6$ (domain score of $<54$ ).

The impact on daily life for two domains ( nausea and vomiting)was calculated as follow "No impact of (nausea and vomiting) on daily life" was defined as an average item score of $\geq 6$ on a seven-point scale (domains score of $\geq 108$ ); while, an impact of nausea and vomiting on daily life was defined as an average item score of $<6$ ( domains score of $<108$ ). Operational design

It included preparatory phase, content validity, pilot study, field work phase " implementation phase and evaluation phase. 


\section{preparatory phase}

This phase started by: extensive reviewing of current, past, local and international related literatures as text books, articles, journals, periodicals and magazines was done and study tools were formulated.

\section{Content Validity \& Reliability}

Content validity was done by five expertise ( two nursing staff) from the medical-surgical nursing field and (three oncology specialists) from the medical field to test relevance of the contents, clarity and comprehensiveness of the tools. Reliability of the FLIE was assessed using Chronbach's Alpha, a measure of inter-item correlation or consistency of response within an instrument. The calculated alpha was above 0.9 for the responses given before and after administrations of the FLIE.

\section{( Lindley et al., 2013).}

\section{Pilot study}

Pilot study was conducted on $10 \%$ of sample in selected setting (5 patients) to evaluate applicability and clarity of the tools, estimate the time needed for data collection, test the feasibility of conducting the research after analyzing the pilot study results, slight modifications were done accordingly. These patients were not included in the actual study .

\section{Ethical consideration}

An official permission was obtained from the head of medical department at Qena health insurance hospital. In addition to verbal permission for voluntary participation was obtained from patients and the nature and purpose of the study were explained.

\section{Fieldwork phase}

- Data were collected during the period from 1/11/ 2017 to $10 / 5 / 2018$.

- The admission days for receiving chemotherapy at Qena health insurance hospital were five days weekly.

- Some patients were first receivers, and others were receiving chemotherapy in the form of cycles which may be every two or three weeks that continue until completing the treatment course.

- At initial interview the researcher introduced herself to initiate line of communication in order to facilitate the implementation of the tools.

- Initial assessment was done for all patients during admission as a base line data by using tool-I ( socio-demographic data, medical data, physical examination, diagnostic investigations) and tool (II).

- Also the researcher reassessed patients on the six $^{\text {th }}$ day after chemotherapy administration (with a five days recall) by using tool-I "some items of part two, part three (Nausea and vomiting grading, nutritional assessment and laboratory investigation) and tool II for all studied sample to distinguish between the initial assessment and reassessment after chemotherapy administration.

\section{Statistical analysis}

Data entry and analysis were done using SPSS ver.20 ( statistical Package for Social Science). data were presented by using the following tests; number, percentage, chi square, mean and standard deviation. A Probability level of 0.05 was adopted as a level of significance for testing the research results. 
Results

Table (1): Frequency distribution of socio-demographic data of studied patients $(n=48)$.

\begin{tabular}{|l|c|c|}
\hline \multicolumn{1}{|c|}{ Socio-demographic data } & N & \% \\
\hline Age groups & & 31.3 \\
\hline $18-\leq 39$ yrs. & 15 & 39.6 \\
\hline $40-\leq 59$ yrs. & 19 & 29.1 \\
\hline$>60$ yrs. & 14 & \\
\hline Mean \pm SD & & $47.12 \pm 14.21$ \\
\hline Sex & 31 & 64.9 \\
\hline Male & 17 & 35.4 \\
\hline Female & & \\
\hline Marital status & 8 & 16.7 \\
\hline Single & 32 & 66.7 \\
\hline Married & 8 & 16.7 \\
\hline Widow & & \\
\hline Residence & 25 & 52.1 \\
\hline Urban & 23 & 47.9 \\
\hline Rural & & \\
\hline Occupation & 27 & 56.3 \\
\hline Employed & 21 & 43.8 \\
\hline Unemployed & & \\
\hline Education level & 6 & 12.5 \\
\hline Illiterate & 2 & 4.2 \\
\hline Elementary & 5 & 10.4 \\
\hline Preparatory & 23 & 47.9 \\
\hline Secondary & 12 & 25.0 \\
\hline University & & \\
\hline Income & 16 & 33.3 \\
\hline Less than 2000p & 32 & 66.7 \\
\hline More than 2000p & & \\
\hline
\end{tabular}

Table (2): Frequency distribution of studied patients according to stages of disease and chemotherapy regimens $(n=48)$.

\begin{tabular}{|l|c|c|}
\hline \multicolumn{1}{|c|}{ Disease stages \& chemotherapy regimens } & N & \% \\
\hline Stage of disease & & 47.9 \\
\hline$\bullet$ Stage (II) & 23 & 52.1 \\
\hline$\bullet$ Stage (III) & 25 & \\
\hline Chemotherapy experience & 37 & 77.1 \\
\hline$\bullet$ Non & 11 & 22.9 \\
\hline$\bullet$ First & & 22.9 \\
\hline Types of chemotherapy regimens & 11 & 20.8 \\
\hline$\bullet 1^{\text {st }}$ group (neoadjective chemotherapy) & 10 & 56.3 \\
\hline$\bullet 2^{\text {nd }}$ group (adjective chemotherapy). & 27 & \\
\hline$\bullet \cdot$ rd group (chemotherapy for advanced cancer). & & 4.2 \\
\hline Frequency of administration & 2 & 56.2 \\
\hline$\bullet$ One week & 27 & 37.5 \\
\hline$\bullet$ Two weeks & 18 & 2.1 \\
\hline$\bullet$ Three weeks & 1 & \\
\hline$\bullet$ Month & & \\
\hline
\end{tabular}

Vol , (7) No, (17) June, 2019 
Table (3): Comparison between studied patients according to nausea and vomiting grading scale before and after chemotherapy $(n=48)$.

\begin{tabular}{|l|c|c|c|c|c|}
\hline Nausea and vomiting grading & \multicolumn{2}{|l|}{ Before chemotherapy } & \multicolumn{2}{l|}{ After chemotherapy } & P.value \\
\hline Nausea grades & $\mathrm{N}$ & $\%$ & $\mathrm{~N}$ & $\%$ & \\
\hline No nausea & 33 & 68.8 & 5 & 10.4 \\
\hline Grade 1 (mild ) & 10 & 20.8 & 15 & 31.2 & \multirow{3}{*}{$001^{* *}$} \\
\hline Grade 2 (moderate ) & 5 & 10.4 & 28 & 58.4 & \\
\hline Grade 3 (sever ) & 0 & 0 & 0 & 0 & \\
\hline Grade 4 (life threatening ) & 0 & 0 & 0 & 0 & \\
\hline Vomiting grades & & & & & \\
\hline No vomiting & 25 & 52.0 & 12 & 25.0 & \multirow{2}{*}{$0.01^{*}$} \\
\hline Grade 1 (mild ) & 12 & 25.0 & 12 & 25.0 & \\
\hline Grade 2 (moderate ) & 10 & 20.9 & 22 & 45.8 & \\
\hline Grade 3 (sever ) & 1 & 2.1 & 2 & 4.2 & \\
\hline Grade 4 (life threatening ) & 0 & 0 & 0 & 0 & \\
\hline
\end{tabular}

Chi-Square Tests $\quad *=$ Significant difference, ${ }^{*} p \leq 0.05 \quad{ }^{* *}=$ highly significance, ${ }^{*} p \leq 0.01$

Ns= Non significant difference

Table (4): Comparison between studied patients before and after chemotherapy according to their anthropometric measurements (body surface area and skin turgor).

\begin{tabular}{|c|c|c|c|c|c|}
\hline Anthropometric measurement & \multicolumn{2}{|c|}{ Before chemotherapy } & \multicolumn{2}{|c|}{ After chemotherapy } & \multirow[t]{2}{*}{ p. value } \\
\hline Body surface $\operatorname{area}(\mathrm{m})^{2}$ & $\mathbf{N}$ & $\%$ & $\mathbf{N}$ & $\%$ & \\
\hline - Low weight $<1.5 \mathrm{~m}^{2}$ & 14 & $29.1 \%$ & 23 & $47.9 \%$ & \multirow{3}{*}{$0.001 * *$} \\
\hline -Standers level of weight $\left(1.5-2 \mathrm{~m}^{2}\right)$ & 30 & $62.5 \%$ & 23 & $47.9 \%$ & \\
\hline -Over weight $\left(>2 \mathrm{~m}^{2}\right)$ & 4 & $8.4 \%$ & 2 & $4.2 \%$ & \\
\hline \multicolumn{6}{|l|}{ Skin turgor test } \\
\hline - Good hydration (Recoils immediately). & 34 & $70.8 \%$ & 30 & $62.5 \%$ & $0.01 * *$ \\
\hline - Mild dehydration $(<2$ second $)$ & 12 & $25.0 \%$ & 14 & $29.1 \%$ & $0.01 * *$ \\
\hline -Moderate dehydration (2-10 second) & 2 & $4.2 \%$ & 4 & $8.4 \%$ & $0.01 * *$ \\
\hline $\begin{array}{l}\text { - Severe dehydration (Several seconds or } \\
\text { minutes). }\end{array}$ & 0 & 0 & 0 & 0 & ----- \\
\hline
\end{tabular}

Chi-Square Tests $\quad *=$ Significant difference, ${ }^{*} p \leq 0.05 \quad * *=$ highly significance, ${ }^{*} p \leq 0.01$

$N s=$ Non significant difference $p>0.05$

Table (5): Comparison between laboratory investigations for studied patients before and after chemotherapy.

\begin{tabular}{|c|c|c|c|c|c|}
\hline \multirow{2}{*}{$\begin{array}{c}\text { Laboratory investigations } \\
\text { Complete blood count } \\
\end{array}$} & \multicolumn{2}{|c|}{ Before chemotherapy } & \multicolumn{2}{|c|}{ After chemotherapy } & \multirow{2}{*}{ p.value } \\
\hline & $\mathbf{N}$ & $\%$ & $\mathbf{N}$ & $\%$ & \\
\hline \multicolumn{6}{|c|}{ WBC ( white blood cells) count } \\
\hline $\begin{array}{l}\text { - Normal (Normal Range) } \\
\text { ( 4,000-11,000 cell per mm3 }\end{array}$ & 23 & 47.9 & 22 & 45.8 & $0.11^{\mathrm{n} . \mathrm{s}}$ \\
\hline $\begin{array}{l}\text { - leukopenia (Low Range) } \\
<4,000 \text { cell per mm3) }\end{array}$ & 19 & 39.6 & 15 & 31.2 & $0.001 * *$ \\
\hline $\begin{array}{l}\text { - leukocytosis ( High Range) } \\
\text { ( > 11,000 cell per mm3) }\end{array}$ & 6 & 12.5 & 11 & 23.0 & $0.001 * *$ \\
\hline \multicolumn{6}{|l|}{ RBC ( red blood cells) count } \\
\hline $\begin{array}{l}\text { - Normal (Normal Range) } \\
\text { (4.5 to } 5.5 \text { million/mcL). }\end{array}$ & 24 & 50.0 & 23 & 48.0 & $0.11 \mathrm{n.s}$ \\
\hline $\begin{array}{l}\text { - Erythropenia (Low Range) } \\
(<4 \text { million/mcL) }\end{array}$ & 22 & 45.8 & 18 & 37.5 & $0.001 * *$ \\
\hline $\begin{array}{l}\text { - polycythemia ( High Range) } \\
\text { ( }>6 \text { million/mcL). }\end{array}$ & 2 & 4.2 & 7 & 14.5 & $0.001 * *$ \\
\hline
\end{tabular}

Vol , (7) No , (17) June, 2019 


\begin{tabular}{|c|c|c|c|c|c|}
\hline Laboratory investigations & \multicolumn{2}{|c|}{ Before chemotherapy } & \multicolumn{2}{|c|}{ After chemotherapy } & \multirow{2}{*}{ p.value } \\
\hline Complete blood count & $\mathbf{N}$ & $\%$ & $\mathbf{N}$ & $\%$ & \\
\hline \multicolumn{6}{|c|}{ Hgb ( Hemoglobin ) } \\
\hline - Hemoglobin level (12 to 16 gm/dL) & \multicolumn{2}{|c|}{$10.53 \pm 3.43$} & \multicolumn{2}{|c|}{$9.85 \pm 3.89$} & $.370^{\mathrm{ns}}$ \\
\hline \multicolumn{6}{|l|}{ Platelets count } \\
\hline - Normal( $150,000-400,000 \mathrm{c} / \mathrm{mcl})$ & 32 & $66.6 \%$ & 32 & 66.6 & \multirow{3}{*}{.022} \\
\hline - thrombocytopenia $(\perp 150,000)$ & 14 & $29.2 \%$ & 11 & 23.0 & \\
\hline -thrombocytosis $(\mathbf{4} 400,000)$ & 2 & $4.2 \%$ & 5 & 10.4 & \\
\hline \multicolumn{6}{|l|}{ Chemistry investigation } \\
\hline \multicolumn{6}{|l|}{ Sodium } \\
\hline - Normal range (135-145mmol/L) & 32 & 66.6 & 28 & 58.3 & \multirow{3}{*}{$0.001 * *$} \\
\hline $\begin{array}{l}\text { - Hyponatremia(Low Range) } \\
(<135 \mathrm{mmol} / \mathrm{L})\end{array}$ & 11 & 23.0 & 4 & 8.4 & \\
\hline $\begin{array}{l}\text { - Hypernatremia ( High Range) } \\
(>145 \mathrm{mmol} / \mathrm{L} \text {. }\end{array}$ & 5 & 10.4 & 16 & 33.3 & \\
\hline \multicolumn{6}{|l|}{ Potassium } \\
\hline - Normal range (3.5 and $5.0 \mathrm{mEq} / \mathrm{L})$ & 30 & 62.5 & 22 & 45.8 & \multirow{3}{*}{$0.002 * *$} \\
\hline $\begin{array}{ll}\text { Hypokalemia } & \text { (LowRange) } \\
(<3.5 \mathrm{mmol} / \mathrm{L}) . & \end{array}$ & 12 & 25.0 & 18 & 37.5 & \\
\hline $\begin{array}{l}\text { Hyperkalemia ( High Range) } \\
(>5.5 \mathrm{mmol} / \mathrm{L})\end{array}$ & 6 & 12.5 & 8 & 16.7 & \\
\hline \multicolumn{5}{|l|}{ Calcium } & \multirow{4}{*}{$0.05 *$} \\
\hline Normal range $\quad(2.12 .6 \mathrm{mmol} / \mathrm{L})$ & 15 & 31.2 & 9 & 18.7 & \\
\hline $\begin{array}{l}\text { hypocalcemia(Low } \text { Range }<2 \\
\text { mmol/L). }\end{array}$ & 12 & 25.0 & 11 & 23.0 & \\
\hline $\begin{array}{lll}\text { Hypercalcemia } & \text { (High } & \text { Range } \\
(>2.6 \mathrm{mmol} / \mathrm{L}) & & \\
\end{array}$ & 21 & 43.8 & 28 & 58.3 & \\
\hline
\end{tabular}

Chi-Square Tests and independent t-test $*=$ Significant difference, $*^{*} \leq 0.05 \quad * *=$ highly significance , $* p \leq 0.01 \quad N s=$ Non significant difference $\quad p>0.05$

Table(6): Comparison between studied patients before and after chemotherapy according to activates unable to do and actions taken due to nausea and vomiting.

\begin{tabular}{|c|c|c|c|c|c|}
\hline Activates and actions due to CINV & \multicolumn{2}{|c|}{ Before chemotherapy } & \multicolumn{2}{|c|}{ After chemotherapy } & p.value \\
\hline \multicolumn{6}{|l|}{ Activities unable to do. } \\
\hline - Take care of self & 13 & 27.1 & 19 & 39.6 & $.001^{* *}$ \\
\hline - Take care of others & 15 & 31.3 & 21 & 43.8 & $.001^{* *}$ \\
\hline - Prepare meal & 7 & 14.6 & 7 & 14.6 & $0.5^{\mathrm{n} . \mathrm{s}}$ \\
\hline - Eat meal & 25 & 52.1 & 27 & 56.3 & $0.5^{\text {n.s* }}$ \\
\hline - Go to work & 24 & 50.0 & 25 & 52.1 & $0.5^{\mathrm{n} . \mathrm{s}}$ \\
\hline - Run errands & 19 & 39.6 & 10 & 20.8 & $.001^{* *}$ \\
\hline - Household tasks & 8 & 16.7 & 8 & 16.7 & $0.5^{\mathrm{n} . \mathrm{s}}$ \\
\hline - Activities with friends & 13 & 27.1 & 5 & 10.4 & $.001^{* *}$ \\
\hline - Take medications & 15 & 31.3 & 14 & 29.2 & $0.5^{\mathrm{n} . \mathrm{s}}$ \\
\hline \multicolumn{6}{|l|}{ Actions taken. } \\
\hline - Called physician & 26 & 54.2 & 23 & 47.9 & $.001^{* *}$ \\
\hline - Visited pharmacy & 9 & 18.8 & 10 & 20.8 & $0.1^{\mathrm{n} . \mathrm{s}}$ \\
\hline -Purchased medications(not-prescribed) & 13 & 27.1 & 16 & 33.3 & $.001^{* *}$ \\
\hline - Purchased special foods & 15 & 31.3 & 13 & 27.1 & $0.1^{\text {n.s }}$ \\
\hline - Delayed chemotherapy & 18 & $37.5 \%$ & 22 & 43.8 & $.001^{* * *}$ \\
\hline
\end{tabular}

Chi-Square Tests $*=$ Significant difference, $* p \leq 0.05 \quad * *=, * p \leq 0.01 \quad$ Ns $=$ Non significant difference $\quad p>0.05$ 
Table (7): Frequency distribution of studied patients before and after chemotherapy according to consequences experienced by them due to CINV.

\begin{tabular}{|c|c|c|c|c|c|}
\hline \multirow[t]{2}{*}{ Consequences of CINV } & \multicolumn{2}{|c|}{ Before chemotherapy } & \multicolumn{2}{|c|}{ After chemotherapy } & \multirow[t]{2}{*}{ p.value } \\
\hline & $\mathbf{N}$ & $\%$ & $\mathbf{N}$ & $\%$ & \\
\hline Dehydration & 6 & 12.5 & 7 & 14.6 & $0.1^{\mathrm{n} . \mathrm{s}}$ \\
\hline - Malnutrition & 4 & 8.3 & 15 & 31.3 & $.001^{* *}$ \\
\hline - Electrolyte imbalance & 14 & 29.2 & 15 & 31.3 & $0.1^{\mathrm{n} . \mathrm{s}}$ \\
\hline - Anorexia & 35 & 72.9 & 19 & 39.6 & $.001^{* *}$ \\
\hline - Depression & 11 & 22.9 & 9 & 18.8 & $0.5^{\mathrm{n} . \mathrm{s}}$ \\
\hline - Confusion and lethargy & 2 & 4.2 & 2 & 4.2 & $0.135^{\mathrm{n} . \mathrm{s}}$ \\
\hline - Tachycardia and Hypotension & 5 & 10.4 & 8 & 16.7 & $.001^{* *}$ \\
\hline - Salivation and Fever & 2 & 4.2 & 9 & 18.8 & $.003^{* *}$ \\
\hline - High antiemetic cost & 0 & 0 & 10 & 20.8 & $0.001^{* *}$ \\
\hline - Stop cancer treatment or premature cessation & 2 & 4.2 & 2 & 4.2 & $0.135^{n . s}$ \\
\hline - Delay treatment & 10 & 20.8 & 16 & 33.3 & $.001^{* *}$ \\
\hline - Anxiety and psychotic disorder & 35 & 72.9 & 4 & 8.3 & $.001^{* *}$ \\
\hline -Incisional hernia or Wound dehiscence & 0 & 0 & 6 & 12.5 & $.013^{*}$ \\
\hline - Aspiration & 0 & 0 & 0 & 0 & --------- \\
\hline •Mallory_Weiss Syndrome & 2 & 4.2 & 15 & 31.3 & $.001^{* *}$ \\
\hline - Decreased nutritional intake & 2 & 4.2 & 0 & 0 & $0.247^{\mathrm{n} . \mathrm{s}}$ \\
\hline
\end{tabular}

Chi-Square Tests $\quad *=$ Significant difference, ${ }^{*} p \leq 0.05$

Non significant difference $\quad p>0.05$

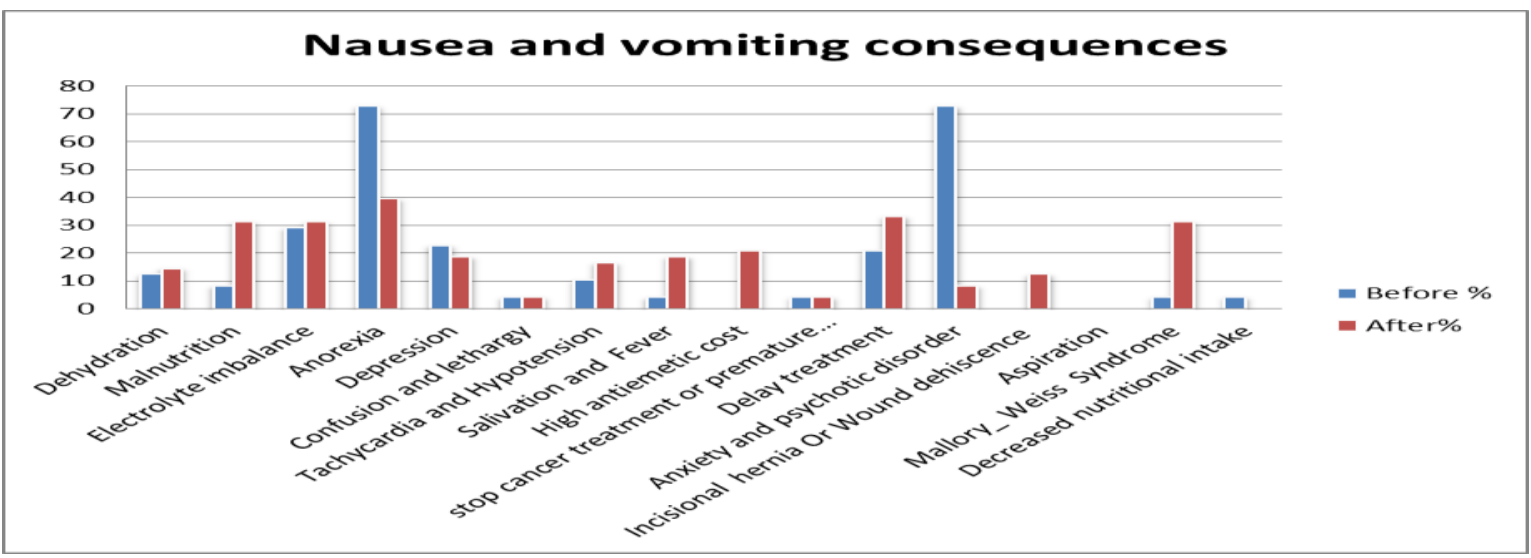

Fig. (1) percentage distribution of consequences due to CINV

Table (8): Comparison of functional daily living for studied patients as measured by FLIE before and after chemotherapy.

\begin{tabular}{|c|c|c|c|c|c|c|c|c|c|}
\hline \multirow{3}{*}{$\begin{array}{l}\text { FLIE of studied } \\
\text { patients }\end{array}$} & \multicolumn{4}{|c|}{ Before chemotherapy } & \multicolumn{4}{|c|}{ After chemotherapy } & \multirow{3}{*}{ p.value } \\
\hline & \multicolumn{2}{|c|}{$\begin{array}{c}\text { No impact } \\
\geq 54\end{array}$} & \multicolumn{2}{|c|}{$\begin{array}{c}\text { Maxim. impact } \\
<54 \\
\end{array}$} & \multicolumn{2}{|c|}{$\begin{array}{c}\text { No impact } \\
\geq 54 \\
\end{array}$} & \multicolumn{2}{|c|}{\begin{tabular}{|c|} 
Maxim. impact \\
$<54$
\end{tabular}} & \\
\hline & No. & $\%$ & No. & $\%$ & No. & $\%$ & No. & $\%$ & \\
\hline $\begin{array}{l}\text { Total score of nausea } \\
\text { domain }\end{array}$ & 1 & 2.1 & 47 & 97.9 & 0 & 0.0 & 48 & 100 & $0.500^{\mathrm{ns}}$ \\
\hline $\begin{array}{l}\text { Total score of vomiting } \\
\text { domain }\end{array}$ & 0 & 0.0 & 48 & 100 & 0 & 0.0 & 48 & 100 & ------ \\
\hline $\begin{array}{l}\text { Total score of FLIE } \\
<108 \text { poor } \\
\geq 108 \text { good }\end{array}$ & 1 & 2.1 & 47 & 97.9 & 0 & 0.0 & 48 & 100 & $0.500^{\mathrm{ns}}$ \\
\hline
\end{tabular}

FLIE ( functional living index emesis). 
Table (9): Relation between consequence of CINV and functional daily living of studied patient.

\begin{tabular}{|c|c|c|c|c|c|c|c|c|c|}
\hline \multirow{3}{*}{ CINV consequences } & \multicolumn{4}{|c|}{ Before chemotherapy } & \multicolumn{4}{|c|}{ After chemotherapy } & \multirow{3}{*}{ p.value } \\
\hline & \multicolumn{2}{|c|}{$\begin{array}{c}\text { Maxim. Impact }< \\
108 \text { (bad } \\
\text { condition) }\end{array}$} & \multicolumn{2}{|c|}{$\begin{array}{l}\text { No impact } \geq \\
108(\text { good } \\
\text { condition) }\end{array}$} & \multicolumn{2}{|c|}{$\begin{array}{l}\text { Maxim. Impact } \\
<108 \text { (bad } \\
\text { condition) } \\
\end{array}$} & \multicolumn{2}{|c|}{$\begin{array}{l}\text { No impact } \geq \\
\quad(\text { good } \\
\text { condition })\end{array}$} & \\
\hline & $\mathbf{N}$ & $\%$ & $\mathbf{N}$ & $\%$ & $\mathbf{N}$ & $\%$ & $\mathbf{N}$ & $\%$ & \\
\hline - Dehydration & 6 & 12.5 & 0 & 0.0 & 7 & 14.6 & 0 & 0.0 & $0.08^{\mathrm{ns}}$ \\
\hline - Malnutrition & 4 & 8.3 & 0 & 0.0 & 15 & 31.3 & 0 & 0.0 & $0.001^{\text {** }}$ \\
\hline Electrolyte imbalance & 14 & 29.2 & 0 & 0.0 & 15 & 31.3 & 0 & 0.0 & $0.08^{\mathrm{ns}}$ \\
\hline - Anorexia & 35 & 72.9 & 0 & 0.0 & 19 & 39.6 & 0 & 0.0 & $0.001^{* *}$ \\
\hline - Depression & 11 & 22.9 & 0 & 0.0 & 9 & 18.8 & 0 & 0.0 & $.0 .001^{* *}$ \\
\hline $\begin{array}{c}\text { Confusion and } \\
\text { lethargy }\end{array}$ & 2 & 4.2 & 0 & 0.0 & 2 & 4.2 & 0 & 0.0 & $0.135^{\mathrm{ns}}$ \\
\hline $\begin{array}{c}\text { Tachycardia and } \\
\text { Hypotension }\end{array}$ & 5 & 10.4 & 0 & 0.0 & 8 & 16.7 & 0 & 0.0 & $0.001^{* *}$ \\
\hline Salivation and Fever & 2 & 4.2 & 0 & 0.0 & 9 & 18.8 & 0 & 0.0 & $.0 .001^{* *}$ \\
\hline - High antiemetic cost & 0 & 0.0 & 0 & 0.0 & 10 & 20.8 & 0 & 0.0 & $.0 .001^{* *}$ \\
\hline $\begin{array}{l}\text { stop cancer treatment } \\
\text { or premature } \\
\text { cessation }\end{array}$ & 2 & 4.2 & 0 & 0.0 & 2 & 4.2 & 0 & 0.0 & $0.135^{\mathrm{ns}}$ \\
\hline - Delay treatment & 10 & 20.8 & 0 & 0.0 & 16 & 33.3 & 0 & 0.0 & $0.001^{\text {** }}$ \\
\hline $\begin{array}{l}\text { Anxiety and } \\
\text { psychotic disorder }\end{array}$ & 35 & 72.9 & 0 & 0.0 & 4 & 8.3 & 0 & 0.0 & $0.001^{* *}$ \\
\hline $\begin{array}{l}\text { Incisional hernia } \\
\text { Or Wound } \\
\text { dehiscence }\end{array}$ & 0 & 0.0 & 0 & 0.0 & 6 & 12.5 & 0 & 0.0 & $.013^{\mathrm{ns}}$ \\
\hline - Aspiration & 0 & 0.0 & 0 & 0.0 & 0 & 0.0 & 0 & 0.0 & --------- \\
\hline $\begin{array}{l}\text { Mallory_Weiss } \\
\text { Syndrome } \\
\end{array}$ & 2 & 4.2 & 0 & 0.0 & 15 & 31.3 & 0 & 0.0 & $0.001^{* *}$ \\
\hline $\begin{array}{l}\text {-Decreased nutritional } \\
\text { intake }\end{array}$ & 2 & 4.2 & 0 & 0.0 & 0 & 0.0 & 0 & 0.0 & $0.247 \mathrm{~ns}$ \\
\hline
\end{tabular}

Table(1): Shows that, The mean age of studied patients was $(47.12 \pm 14.21)$, more than half of studied patients $(64.9 \%)$ was male, from urban area, employed and married $(52.1 \%, 56.3 \%$ and $66.7 \%$ respectively). According to educational and economic status, less than half of patients(47.9\%) had secondary education and more than half of them $(66.7 \%)$ their monthly income was more than 2000p.

Table (2): Revealed that, more than half of studied patients $(52.1 \%)$ had diagnosed in stage (III) of the tumor, had received chemotherapy for advanced cancer per two weeks $(56.3 \%$, and56.2\% respectively). Majority of patients $(77.1 \%)$ were nonfirst receivers for chemotherapy.

Table (3): Shows that, There were significant differences between studied patients according to nausea and vomiting grading scale, in which there were obvious increase in rate of nausea and vomiting especially grade (II) for studied patients after chemotherapy than before .

Table (4): Shows that, There were statistical significance differences between studied patients before and after chemotherapy according to their body surface area. as two thirds of studied patients $(62.5 \%)$ had standards level of weight before chemotherapy, while more than of one third of studied patients $(47.9 \%)$ converted from standard level of weight to low weight after chemotherapy. Also we observe from this table; there were statistical significant changes in skin turgor tests for studied patents after chemotherapy than before as about one third $(29.1 \%)$ of studied patients suffered from mild dehydration after chemotherapy in spite of majority of them(70.8\%) had good hydration before chemotherapy.

Table (5): Reveals that, there were highly statistical significant changes in WBC and RBC counts before and after chemotherapy. Also there were significant deteriorations of blood chemistries among studied patients after chemotherapy than before.

Table (6): Shows that, There were statistical significant increase in number of patients who unable to do some activities after chemotherapy than they are used to do it before chemotherapy. Also there 
were statistical significant increases in costs due to the actions that those patients taken it after receiving chemotherapy.

Table (7) \& figure (1): Reveal that, majority of studied patients $(72.9 \%)$ had suffered from anorexia, anxiety and psychotic disorder before chemotherapy whose decreased after chemotherapy. About one third of studied patients $(31.3 \%)$ had experienced malnutrition, electrolyte imbalance and delayed treatment after they had received chemotherapy.

Table (8): Shows that, there were no statistical significant differences between studied patients either before or after chemotherapy according to their functional daily living as measured by FLIE. Also this table shows that CINV had maximum impact on nearly all patients' functional daily living.

Table (9): Reflects that; no one of patients had good condition of functional daily living either before or after chemotherapy. Also we observe from this table that about one third or less of studied patients suffered from consequences as malnutrition, electrolyte imbalance, Mallory_ Weiss Syndrome $(31.3 \%)$ delayed treatment, salivation and fever, high antiemetic cost, ( $33.3 \%, 18.8 \%, 20.8 \%$ respectively) which increased after chemotherapy than before among patients who had bad condition of functional daily living. Also all consequences were apparent among patients who had bad functional daily living.

\section{Discussion}

The present study showed that ; More than half of studied patients were male, Their mean age was forty- seven years old. from urban area, employed, married, had secondary education and had limited month salary. These study findings are in accordance with American Institute for Cancer Research, (2017) that published that $\mathrm{CRC}$ is ranked among the top ten cancers occurring in males and females aged twenty- forty nine years old. Persons younger than fifty years old were more likely to present with less localized and more distant disease than do older adults. Also current results agree with a study conducted by Murphy et al., (2017)which showed that ; CRC incidence rates are approximately thirty percent higher in men than women.

Another study conducted by Wood et al., (2016) which arrived to all cancer places combined, residents of poorer counties have thirteen percent higher death rates from cancer in men and 3\% higher rates in women compared with more affluent counties. In contrast to our findings a study applied by (Ezat, 2014) at National Cancer Institute which revealed that more than ninety percent of people with colorectal cancer are diagnosed after age fifty. the average age at diagnosis was seventy two.
As regard to colorectal stages and treatment; Our study revealed that, more than half of studied patients had diagnosed in stage (III) and less than half of them had diagnosed in stage (II) of the tumor, and two thirds of them had performed colorectal surgery and were receiving advanced chemotherapy. This study result was agreeing with a study conducted by Andre et al., (2013) who stated that sixty percent of colorectal cancer patients who received advanced chemotherapy had diagnosed in stage III colon cancer.

As regard to types of chemotherapy regimens that patients received it in our study; the results of the present study showed that, eleven patients with stage (II) and (III) received first group (neoadjuvant chemotherapy) preoperativly-five of them had received CAPOX (capecitabine + oxaliplatin), and four had received FOLFOX (5-FU, leucovorin+ oxaliplatin), these medications administered in six cycles per three weeks for three months. In addition to, two of them had received (irinotecan, 5-FU and leucovorin) weekly for two months.

The second group of chemotherapy was (adjuvant chemotherapy) that ten patients in our study received it, nine of them had received FOLFOX plus Xeloda ${ }^{\circledR}$ (capecitabine), except one patient in this group had received FOLFOX alone, this group of medications administered in twelve cycles per three weeks for six months. The last type of chemotherapy regimens was the third group that was administered for twenty-seven patients in our study, ten of them received FOLFOX plus Avastin (Bevacizumab), and seventeen of them were received FOLFOX plus Irinotecan (Camptosar), they were administered six cycles through three months (cycle/ fourteen days).

A study by Uehara et al., (2017) published at Journal of Clinical Oncology which reported that, oxaliplatin combined with5_ Fluorouracil and Leucovorin as a standard surgical adjuvant chemotherapy for stage II and III colon cancer. In addition Toirinotecan and Bevacizumab in advanced cancer as a first line of treatment for metastasis.

As regarding to nausea and vomiting grades due to chemotherapy. Half of studied patients in the present study had moderate nausea and vomiting (grade II) after chemotherapy, In spite of more than half of them didn't have either nausea or vomiting before chemotherapy. These could be explained by that these patients were receiving palliative treatment before chemotherapy such as (Ranitidine, Dexamesathone, and Zofran) which led to its less occurrence before than after. This result finding was disagreeing with a study conducted by BC Cancer Agency, (2017) which explained that, Not all chemotherapy drugs cause nausea and vomiting. But Individual drugs vary in their effects, These 
symptoms are also more likely to happen when combinations of chemotherapy drugs are given.

As regarding to nutritional assessment ( body surface area and skin turgor ), The findings of our study illustrated that, there was highly statistical significant difference between studied patients before and after chemotherapy according to BSA ( body surface area). More than one third of studied patients converted from standard level of weight before chemotherapy to low weight and mild dehydration. Also there were significant changes in skin turgor tests for studied patients after chemotherapy than before as the number of patients who suffered from dehydration was higher than before.

Murry et al., (2018) stated that, Nutrition is an important part of cancer treatment and eating the right kinds of foods during and after chemotherapy regimen can help cancer patients to be feel better and stay stronger.

Similar to our results, Ayala et al., (2016) who reported that, LE ( low energy) diet demonstrated significant reductions in body weight, plasma, albumin and visceral fat.

As regard to laboratory investigations (complete blood count and blood chemistries). The current study showed a highly statistical significant changes in both hemoglobin level, white and red blood cells counts, in which more than one third of studied patients experienced leukopenia, erythropenia and low hemoglobin level before and after chemotherapy. While one third of studied patients experienced hypernatremia, hypokalemia and hypercalcemia regarding to chemistry investigation results. This lab findings may reflect the intensity of chemotherapy adverse effects on circulatory status for our studied sample

Schueren,(2015) explained that chemotherapy is the most common cause of neutropenia, which kills rapidly reproducing cells including healthy white cells. Also there was in the same line with our study, another study conducted by Anna et al., (2018) who found that cancer can affect red blood cell levels, resulting in anemia due to inflammatory cytokines released by the tumor or the therapy, decreased erythropoitenproduction in the kidneys, or impaired iron utilization. The present study lab findings agree with a study applied by (Ahmed, 2016) which conducted at general health and nutrition department of El mansoura University Hospital which revealed that cancer patients can develop abnormal electrolyte levels, such as hypernatremia related to dehydration and high liver enzymes.

According to activities and the actions taken by studied patients due to CINV ; our study results revealed that, more than half of patients refused eating and unable to go to their work before and after chemotherapy and about one third of patients were unable to run errands, take care of others, take care of self and take additional medication before and after chemotherapy, all of this led patients to take actions to restore their strength or balance their functional daily living, as our results showed that half of patients called with their physicians before and after chemotherapy and about one third of them delayed their chemotherapy regimens, purchased special foods and non prescribed medications before and after chemotherapy .

Similar to our study findings a study contacted by Yabroff et al., (2016) who found that; the economic impact associated with twenty-three percent of patients who experienced emesis being unable to go to work and twenty-two percent reported that they were unable to prepare meals or take care of themselves due to emesis. in the same line with our study, a study conducted by Zafar et al., (2015) which revealed that; the high costs of cancer care have been associated with lower adherence to medications, serious material and psychological financial hardship.

According to various consequences that had experienced by studied patients due to CINV, our study revealed that; a significant association between CINV and consequences acceleration after chemotherapy than before. This could be due to the effect of chemotherapy. Versus that occurred with anorexia, anxiety and psychotic disorders which increased before chemotherapy than after. The rationale for this difference may be due to the previous negative experience of these patients with chemotherapy.

In the same line with our study Schwartzberg et al., (2015) arrived to that CINV not only is distressing for the patient , but it also can lead to dehydration, electrolyte imbalances, weight loss, and malnourishment, resulting in additional office visits, emergency department (ED) visits, or hospitalizations, thus requiring additional supportive care therapies. This increase in resource utilization increases the overall cost of cancer care. Furthermore, dose reductions or delays in chemotherapy may be necessary as a result of CINV, which negatively impact on patient outcomes .

Another study reported by Rhodes \& McDaniel, (2017) who explained that, nausea and vomiting have a considerable impact on all aspects of the patients' qualities of life, as well as those of their family and caregivers. The distress resulting from these symptoms can escalate over time and can potentially lead to a patient's refusal to continue with the most effective antitumor therapy . Indeed, failure to control these side effects can lead to $25 \%-50 \%$ of patients 
delaying or refusing possible life saving antineoplastic therapy .

Recently, Norman et al., (2018) proved that a cytotoxic agents potentially eradicate or reduce tumor size, but may have several toxic side effects that in turn can weaken the patient, particularly by decreasing appetite or inducing emesis, fatigue and asthenia. When malnutrition establishes impairs the immune status and reduces the body's defense against infectious diseases .

Technical University of Munich (TUM), (2018)explained that, malnutrition as a consequence of CINV can increase the incidence of postoperative complications, such as delayed wound healing, dehiscence of anastomosis, morbidity, and mortality, it can be necessary to reduce the dose of cytotoxic agents and/or modify the chemotherapy timing between temporary or definite cessation of treatment. According to functional daily living, our study revealed that there was a maximum impact on functional daily living due to CINV for studied patients, as there were a mild decline in functional daily living for studied patients, either before or after chemotherapy. This declining in patents' functional daily living may be due to external stressors that patients were suffering from it such as (personal, familial, social, financial, psychological problems, and administrative routine that facing it in hospital).

Selyes, (2014) stated that a non cancer life stressors can substantially impact long -term of functional living for cancer patients, in addition to medical variables such as chemotherapy and cancer stage, thus highlighting the importance of evaluating the stress burden on patients in ongoing cancer care.

As regard to relation between CINV consequences and functional daily living of studied patients. The present study results revealed that; no one had good condition of functional daily living either before or after chemotherapy, But worsen after chemotherapy than before among patients who had bad condition of functional daily living previously.

Our study findings in the same line with a study that conducted by Lachaine et al., (2015) who found that, a strong negative impact on patients DA (daily activities) and QoL (quality of life) with both the presence of significant nausea or vomiting as well as their duration. Also in the same context Osoba et al., (2016)mwho stated that CINV has a broad range of consequences that can affect not only patient's general health status but also on their daily functions and quality of life.

\section{Conclusion}

We can conclude that; All patients suffered from consequences due to CINV in varied degrees that may be either before or after chemotherapy as malnutrition, electrolyte imbalance, delayed treatment, Mallory_ Weiss syndrome, salivation and fever, high antiemetic cost, anorexia, tachycardia and hypotension, depression, dehydration, incisional hernia or wound dehiscence, anxiety and psychotic disorders. which reflected on their functional daily living as most of studied patients had worse condition before chemotherapy and all of them had worse condition of functional daily living after chemotherapy too.

\section{Recommendation}

Colorectal cancer patients who receive chemotherapy in need to intensive nursing care and appropriate strategies to manage chemotherapy side effects and consequences related to these side effects, which can improve their functional daily living.

\section{References}

1. Ahmed F., (2016): Relationship between quality of diet and quality of life for cancer patient at general health and nutrition department in medical faculty at Elmansoura University: Issue (9) P.p.79-92.

2. American Institute for Cancer Research. Available at: http:// www.aicr.org. Accessed on September 7., (2017)

3. Andre' T., Sargent D., Tabernero J., (2013): Current issues in adjuvant treatment of stage II colon cancer. Ann Surg.Oncol.vol.13, P.p.887898.

4. Ayala M., Racotta R., Hernández-Montes H., Quevedo L., (2016): Some metabolic effects on lactating rats of a low-energy diet restricted in good-quality protein. Br J Nutr. ; Issue (96) ,P.p. 667-673

5. BC Cancer Agency (BCCA). (2017): Protocol Summary for Adjuvant Combination Chemotherapy for Stage III and Stage IIB Colon Cancer and associated physical and social problems, P.p.77-79; available at: www.bccancer.bc.ca

6. Cohen L., de Moor C., Eisenberg P., (2017): Chemotherapy-induced nausea and vomiting: incidence and impact on patient quality of life at community oncology settings. Support Care Cancer. ; vol. (15) P.. 497-503 .

7. El-Attar, (2015): Cancer statistics, NCI, 2014, Department of Biostatics and Epidemiology, NCI, Cairo, Egypt, available at http://www.nci.cu.edu.eg/lectures/NCI2014.ppt

8. Ezat E., (2014).: Department of Health and Human Services, Centers for Disease Control and Prevention .National Cancer Institute, vol.(2), P. p.196-204. 
9. Hesketh P., (2018): Chemotherapy-induced nausea and vomiting. N Engl J. Med. Vol.(358), P:p.2482-2494.

10. Lachaine J., Yelle L., Kaizer L., Dufour A., Hopkins S., Deuson R., (2015): Chemotherapyinduced emesis: quality of life and economic impact inthe context of current practice in Canada. Support Cancer, vol.( 2), P.p.181-187

11. Lindley C., Hirsch J., \& O'Neill C., (2013): Quality of life consequences of chemotherapyinduced emesis. Qual Life Res 1:331-340.

12. Murphy N., Strickler H., Stanczyk F., (2017): Evaluation of Endogenous Sex Hormone Levels and ColorectalCancer Risk in Postmenopausal Women. J Natl Cancer Inst. Issue.(7) P.p. 93-107.

13. Murry D., Riva L., Poplack D., (2018): Impact of nutrition on pharmacokinetics of antineoplastic agents. Int. J Cancer.vol.(11),P.p.48- 51

14. Norman K., Pichard C., Lochs H., Pirlich M., (2018): Prognostic impact of disease-related malnutrition. ClinNutr.vol. (27), P.p.5-15.

15. Osoba D., Zee B., \& Warr D., (2016): . Effect of post chemotherapy nausea and vomiting on health related quality of life. The quality of life and symptoms control committees of the national cancer institute of Canada clinical trials Group. Support care cancer. Chap. (5) P.p. 307-313.

16. Pickhardt P., Kim D., Pooler B., (2017): Assessment of volumetric growth rates of small colorectal polyps with CT colonography: a longitudinal study of natural history. Lancet Oncol; vol.(14), P.p. 711-720.

17. Rhodes V., \& McDaniel R., (2017): Nausea, vomiting, and retching: complex problems in palliative care. CA Cancer J Clin., vol (51), P.p. 232-248.

18. Robinson J., Callister L., \& Berry J., (2018): Patient centered care and adherence: definitions and applications to improve outcomes. Jam Acad Nurse Pract.; P.p. 600-607.

19.Schueren M., (2015): Nutritional support strategies for malnourished cancer patients. Eur J OncolNurs, vol.(9), Issue.(2), P.p.S74-83

20. Schwartzberg L., Harrow B., Lal L., (2015): Resource utilization for chemotherapy-induced nausea and vomiting events for patients with solid tumors treated with antiemetic regimens. Am Health Drug Benefits. Vol. (8) , Issue.(5) P.p. 273-282.

21. Selyes H., (2014): The stress of life, Assessing the impact of chemotherapy induced nausea and vomiting on patients' daily life, volume(11) P.p. 522-527.

22. Technical University of Munich (TUM): available at: https://www.tum.de/en/about- tum/news/press-releases, Accessed on September 18, (2018).

23. Uehara K., Hiramatsu K., Maeda A., (2017): Neoadjuvantoxaliplatin and capecitabine and bevacizumab without radiotherapy for poorrisk rectal cancer: N-SOG 03 Phase II trial. Jpn J ClinOncol ;volume (43).chap. (9), P.p. 64-71.

24. Wood J., \& Eilers J., (April 2016): Nausea and vomiting in the hematopoietic stem cell transplant population. Poster presented at the State of the Art Nursing Conference; Omaha, Nebraska.vol. 3, P.p.17-21

25. Yabroff K., Dowling E., Guy G., (2016): Financial Hardship Associated With Cancer in the United States: Findings From a Population-Based Sample of Adult Cancer Survivors. J Clin.Oncol. Issue. (34), P.p. 259-267.

26. Zafar S., Peppercorn J., Schrag D., (2015): The financial toxicity of cancer treatment: a pilot study assessing out-of-pocket expenses and the insured cancer patients' experience. Oncologist. Issue.(18) P.p.381-390. 